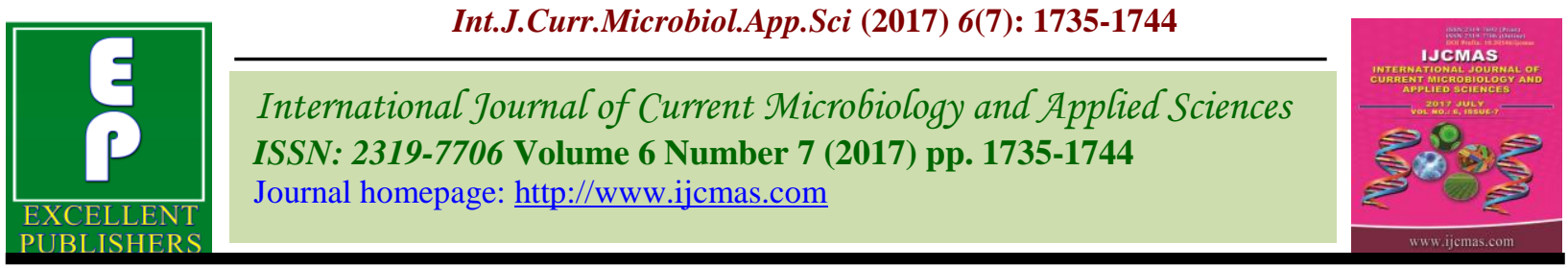

Original Research Article

https://doi.org/10.20546/ijcmas.2017.607.209

\title{
Line x Tester Analysis in Sesame (Sesamum indicum L.)
}

\author{
Kanak Saxena* and Rajani Bisen \\ Department of Genetics and Plant Breeding, Jawaharlal Nehru Krishi Vishwa Vidyalaya, \\ Jabalpur -482026 India \\ *Corresponding author
}

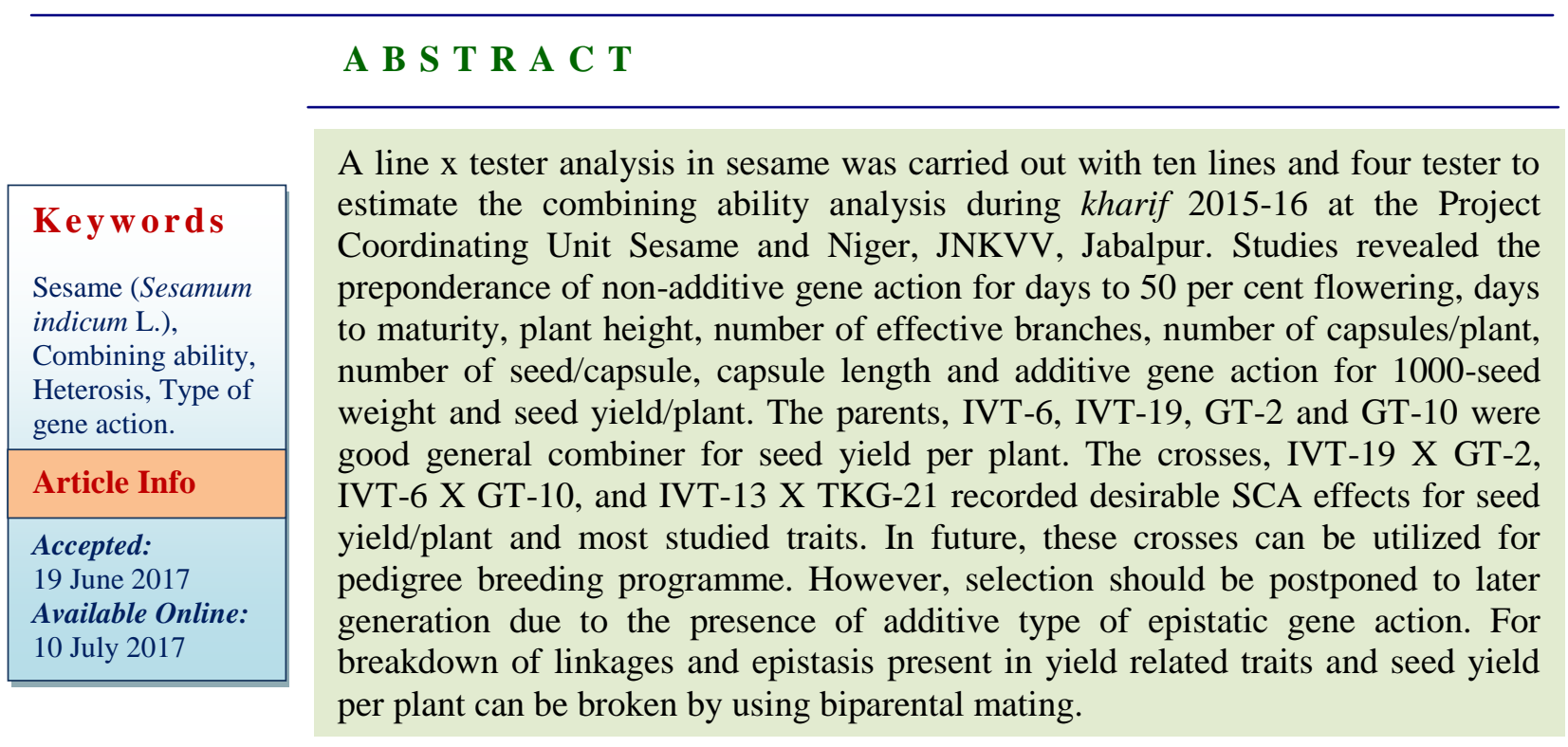

\section{Introduction}

Sesame (Sesamum indicum L.) is a crop, which is cultivated in diverse agroecological situations. It is called as the "Queen of oil seeds" because of its excellent qualities of the seed, oil and meal. Sesame is highly nutritive (oil $50 \%$, protein $25 \%$ ) and its oil contains an antioxidant called sesamol which imparts a high degree of resistance against oxidative rancidity. It is an important annual oilseed crop in the tropics and warm subtropics, where it is usually grown in small patches (Bedigian and Harlan, 1986). India holds a premier position in the global oilseeds scenario accounting for 29 per cent of the total area and 26 per cent of production.
Globally, China and India are the major sesame producers. Rajasthan, Gujarat, Madhya Pradesh, Andhra Pradesh, West Bengal and Tamil Nadu put together constitutes nearly 72 per cent of total area and 58 per cent of total production of sesame in the country. The average productivity is very low as compare to other sesame growing countries and almost stagnant during last few years. In India, sesame is cultivated in 19.53 lakh hectare with a production of 8.32 lakh tonnes and productivity of $426 \mathrm{~kg} / \mathrm{ha}$ (Anonymous, 2015-16). West Bengal, Gujarat, Madhya Pradesh, Rajasthan and U.P are the major sesame growing states. Madhya 
Pradesh contributes $19.71 \%$ and $23.68 \%$ share of country's area (3.85 lakh ha) and production (1.97 lakh tonnes), respectively with productivity $540 \mathrm{Kg} / \mathrm{ha}$. Development of short duration varieties in sesame is gaining importance due to their use as rice fallow crop, catch crop or relay crop. Apart from their wider use, they have several advantages like they require less crop management period, permits multiple cropping system, reduces overall production cost and allows escape from terminal drought. For breaking the present yield barrier and evolving varieties with high yield potential, it is desirable to combine the genes from genetically diverse parents. The success in identifying such parents mainly depends on the gene action that controls the trait under improvement, combining ability and genetic architecture. So far, only one short duration variety, VRI 1 with 75 days duration has been released in Tamil Nadu, Moreover, reports related to early maturity in sesame are very scanty.

There are several techniques for evaluating the varieties or cultivars or lines in terms of their combining ability and genetic architecture. Diallel, partial diallel and line X tester techniques are in common use. Among these, Line $\mathrm{x}$ Tester analysis technique is more suitable for large number of genotypes for understanding the genetical basis at population level (Kempthorne, 1957).

An added advantage of this method is that it gives an overall genetic picture of the materials under investigation in a single generation. In a crop like sesame due to epipetalous flower structure there is good scope for exploitation of heterosis. Further, an understanding of the combining ability and gene action is a prerequisite for any successful hybridization programme. Therefore, the present study was carried out with a view to understand the type of gene action controlling yield and other traits, to identify the best general combiners and the best combinations on the basis of their estimates of general and specific combining ability and to estimate the percentage of heterosis for yield, its attributes through line $\mathrm{x}$ tester analysis in sesame.

\section{Materials and Methods}

The current research work was done at at the Project Coordinating Unit Sesame and Niger, JNKVV, Jabalpur, during successive Kharif seasons 2015 and 2016. Fourteen genetically diverse sesame genotypes comprised of ten lines viz., IVT-1, IVT-6, IVT-8, IVT-13, IVT-14, IVT-15, IVT-16, IVT-18, IVT-19, IVT-20 and four tester viz., TKG-21, TKG22, GT-2 and GT-10 were used in this study. In 2015 season, a line $\mathrm{x}$ tester cross set was done among the fourteen parents, using hand emasculation and pollination as described by Yermanos (1980).

The resulting $40 \mathrm{~F}_{1}$ hybrids along with their parental genotypes were evaluated in layout of randomized complete block with three replications during 2016 season. The experimental unit was raised in single row of four meter length.

Observations were recorded on three randomly selected plants for all the characters viz., days to 50 per cent flowering, days to maturity, plant height, number of primary branches per plant, number of secondary branches per plant, number of capsules/ plant, number of seeds per capsule, 1000-seed weight, harvest index, oil content and seed yield/ plant. The data obtained for each character were analysed by the usual standard statistical procedure (Panse and Sukhatme, 1978). The variation among the hybrids was partitioned further into sources attributed to general combining ability and specific combining ability components in accordance 
with the procedure suggested by Kempthorne (1957). Heterosis was estimated as the deviation of $F_{1}$ mean from mid parent (MP) and better parent (BP) and expressed as percentage.

\section{Results and Discussion}

The analysis of variance for combining ability analysis indicated the presence of significant differences among line $\mathrm{x}$ tester interaction for all the characters (Table 1). The mean sum of square due to males and female were significant for all the traits while, testers showed significant differences for all characters except days to flowering, number of capsules per plant and harvest index indicating the presence of sufficient variability among parents. Presence of significance in parents' vs crosses provided adequacy for comparing the heterotic expression for seed yield/plant and its component traits.

Bharathi Kumar et al., (2009) reported significant differences among parents and hybrids for days to 50 per cent flowering, plant height, number of capsules/ plant and seed yield/plant and non-significant differences for 1000-seed weight. GCA and SCA variance revealed the preponderance of non-additive gene action for days to 50 per cent flowering, days to maturity, plant height, number of primary branches per plant, number of secondary branches per plant, number of capsules per plant, harvest index, oil content and seed yield per plant and additive gene action for number of seeds per capsule and 1000-seed weight.

Similar results for the presence of nonadditive gene action were also reported by Yamanura et al., (2009) for days to 50 per cent flowering, 1000-seed weight and Manivannan and Ganesan (2001), Senthil Kumar and Ganesan (2002), Mothilal et al., (2003), Kumar et al., (2004), Mothilal and
Manoharan (2005), Vidhyavathi et al., (2005), Solanki and Singh (2006), Sharmila and Ganesh (2008) and Bharathi Kumar and Vivekanandan (2009), Mishra and Sikarwar (2001) for days to maturity, capsules/plant and seed yield/plant.

\section{Combining Ability Analysis}

The analysis of variance for combining ability revealed that general combining ability (GCA) variances for females were significant for plant height, number of primary branches per plant, number of secondary branches per plant, number of capsules per plant, number of seeds per capsule, yield per plant, harvest index and oil content On the other hand, specific combining ability (SCA) variances for $\mathrm{f} x \mathrm{~m}$ interaction were highly significant for all the characters except 1000-seed weight.

The magnitude of GCA variances was lower than SCA variances for all the characters indicating the predominance of non-additive gene action. This was further supported by low magnitude of GCA/SCA ratios. The findings are in confirmation with Sakila et al., (2000), Arulmozhi et al., (2001), Manivannan and Ganesan (2001), Mishra and Sikarwar (2001), Senthil Kumar and Ganesan (2002), Mothilal et al., (2003), Kumar et al., (2004), Mothilal and Manoharan (2005), Vidhyavathi et al., (2005), Solanki and Singh (2006), Sharmila and Ganesh (2008) and Bharathi Kumar and Vivekanandan (2009) found that most of the genetic variance for plant height, number of capsules/plant, yield/plant was due to additive gene action.

However, for 1000-seed weight they observed that non-additive variance was the main contributor to genetic variance. Kar et al., (2000) observed that additive genetic variance was predominant for days to maturity, while for days to $50 \%$ flowering, capsules/ plant, capsule length and seed yield/plant, non- 
additive gene action was important. Saravanan and Nadarajan (2003) found that additive genetic variance was of greater importance for days to $50 \%$ flowering, plant height, 1000-seed weight, oil content and harvest index, while non-additive genetic variance appeared with a major role for number of capsules/plant, number of seeds/capsule and single plant yield.

The estimates of general combining ability effects of the parents (Table 3) revealed that the line IVT-6, IVT-19, GT-2 and GT-10 were good general combiner for seed yield per plant and the tester GT-2 and GT-10 showed high gca for seed yield per plant. In addition to seed yield, the line IVT-6 also showed high gca for capsule length and IVT19 showed high gca for number of primary branches, number of secondary branches and 1000-seed weight. While, the tester GT-2 also showed high gca for capsule length and GT-
10 also showed high gca for number of primary branches and days to maturity. These parents could be used in the breeding programme to improve seed yield and its component characters.

It may be inferred that the genotypes can maintain their superiority in per se performance and also combining ability effects. However, the parents that showed superior per se performance for seed yield and its component characters could not express high gca effects for the respective character.

It indicates the poor association between per se performance and gca effects of parents for yield component characters. This was also reported by Ranjith Rajaram et al., (2011) and Patel et al., (2005). Such an absence of parallelism may be due to epistatic interactions.

Table.1 Analysis of variance for combining ability of various quantitative characters

\begin{tabular}{|c|c|c|c|c|c|c|c|c|c|c|c|c|c|}
\hline SOURCE & DF & $\begin{array}{c}\text { Days to } \\
50 \% \\
\text { flowering }\end{array}$ & $\begin{array}{c}\text { Plant } \\
\text { height } \\
(\mathbf{c m})\end{array}$ & $\begin{array}{c}\text { No. of } \\
\text { primary } \\
\text { branche } \\
\text { s per } \\
\text { plant }\end{array}$ & $\begin{array}{c}\text { No. of } \\
\text { secondary } \\
\text { branches } \\
\text { per plant }\end{array}$ & $\begin{array}{l}\text { No. of } \\
\text { capsules } \\
\text { per plant }\end{array}$ & $\begin{array}{l}\text { Capsule } \\
\text { length } \\
(\mathrm{cm})\end{array}$ & $\begin{array}{l}\text { Days to } \\
\text { maturity }\end{array}$ & $\begin{array}{c}\text { No. of } \\
\text { seeds per } \\
\text { capsule }\end{array}$ & $\begin{array}{c}1000 \\
\text { seed } \\
\text { weight } \\
(\mathrm{g})\end{array}$ & $\begin{array}{c}\text { Seed } \\
\text { yield per } \\
\text { plant }\end{array}$ & $\begin{array}{l}\text { Harvest } \\
\text { index (\%) }\end{array}$ & $\begin{array}{c}\text { Oil } \\
\text { conten } \\
\mathbf{t}(\%)\end{array}$ \\
\hline $\begin{array}{l}\text { REPLICATI } \\
\text { ON }\end{array}$ & 2 & 19.43 & 74.03 & 0.01 & 0.32 & 12.32 & $0.02 *$ & 8.57 & $146.16^{* *}$ & 0.02 & $0.23^{* *}$ & 4.77 & 0.06 \\
\hline LINES & 9 & $42.12 * *$ & $782.45 * *$ & $10.36^{* *}$ & $3.88 * *$ & $342.74 * *$ & $0.47 * *$ & $237.75^{* *}$ & $204.33 * *$ & $0.71 * *$ & $31.36^{* *}$ & $164.70^{* *}$ & $\begin{array}{c}6.64 * \\
*\end{array}$ \\
\hline TESTERS & 3 & 3.47 & $348.63^{* *}$ & $7.88^{* *}$ & $1.23 * *$ & 5.66 & $0.15^{* *}$ & $52.74 * *$ & $52.10 *$ & $0.28 * *$ & $0.95 * *$ & 26.21 & $3.95^{*}$ \\
\hline $\begin{array}{c}\text { LINES X } \\
\text { TESTERS }\end{array}$ & 27 & $13.38^{* *}$ & $341.37 * *$ & $5.97 * *$ & $1.40 * *$ & $132.80^{* * *}$ & $0.22 * *$ & $139.21^{* *}$ & $74.98^{* *}$ & $0.10^{*}$ & $0.95 * *$ & $65.54 * *$ & $\begin{array}{c}3.61 * \\
*\end{array}$ \\
\hline ERROR & 78 & 3.70 & 28.41 & 0.09 & 0.11 & 10.73 & 0.01 & 7.84 & 18.25 & 0.06 & 0.02 & 10.66 & 1.11 \\
\hline$\sigma_{\mathrm{f}}^{2}$ & & $2.39 * *$ & $36.76^{* *}$ & $0.37 * *$ & $17.50^{* *}$ & $0.02 * *$ & $8.21 * *$ & $10.78^{* *}$ & $0.05 * *$ & $2.53 * *$ & $8.26 * *$ & $0.25 * *$ & $\begin{array}{c}0.16^{*} \\
*\end{array}$ \\
\hline$\sigma^{2} \mathrm{~m}$ & & -0.33 & $0.24 * *$ & $0.06^{* *}$ & -4.24 & $0.00 * *$ & $-2.88^{* *}$ & $-0.76^{*}$ & $0.01 * *$ & $0.00 * *$ & -1.31 & $0.01 *$ & -0.02 \\
\hline$\sigma_{\mathrm{gca}}^{2}$ & & 0.45 & $10.67^{* *}$ & $0.15^{* *}$ & 1.97 & $0.02 * *$ & $0.29 * *$ & $2.53^{*}$ & $0.02 * *$ & $0.72 * *$ & 1.42 & $0.08^{*}$ & 0.03 \\
\hline$\sigma^{2} \mathrm{sca}$ & & $3.23 * *$ & $104.32 * *$ & $1.96^{* *}$ & $40.69 * *$ & $0.07 *$ & $43.79 * *$ & $18.91^{* *}$ & $0.01 * *$ & $0.31 * *$ & $18.30^{* *}$ & $0.83 * *$ & $\begin{array}{c}0.12 * \\
*\end{array}$ \\
\hline$\sigma_{\mathrm{sca}}^{2} /$ & & 0.14 & 0.10 & 0.08 & 0.05 & 0.26 & 0.01 & 0.13 & 2.36 & 1.35 & 0.08 & 0.10 & 0.27 \\
\hline
\end{tabular}


Table.2 Per se performance of parents for various quantitative characters

\begin{tabular}{|c|c|c|c|c|c|c|c|c|c|c|c|c|c|}
\hline No. & Parents & $\begin{array}{c}\text { Days to } \\
50 \% \\
\text { flowering }\end{array}$ & $\begin{array}{c}\text { Plant } \\
\text { height } \\
\text { (cm) }\end{array}$ & $\begin{array}{c}\text { No. of } \\
\text { Primary } \\
\text { branches } \\
\text { per plant }\end{array}$ & $\begin{array}{c}\text { No. of } \\
\text { Secondary } \\
\text { branches } \\
\text { per plant }\end{array}$ & $\begin{array}{c}\text { No. of } \\
\text { Capsule } \\
\text { per plant }\end{array}$ & $\begin{array}{c}\text { Capsule } \\
\text { length } \\
(\mathrm{cm})\end{array}$ & $\begin{array}{l}\text { Days to } \\
\text { maturity }\end{array}$ & $\begin{array}{c}\text { Seeds } \\
\text { per } \\
\text { capsule }\end{array}$ & $\begin{array}{c}1000 \\
\text { seed } \\
\text { weight } \\
(\mathrm{g}) \\
\end{array}$ & $\begin{array}{l}\text { Yield per } \\
\text { plant (g) }\end{array}$ & $\begin{array}{c}\text { Harvest } \\
\text { index }\end{array}$ & $\begin{array}{c}\text { Oil } \\
\text { content } \\
(\%)\end{array}$ \\
\hline & TKG-21 & 40.67 & 73.8 & 3.56 & 2.81 & 81.96 & 2.85 & 109.33 & 50.84 & 3.28 & 8.85 & 30.15 & 48.93 \\
\hline & TKG-22 & 42.67 & 93.33 & 4.67 & 3.51 & 82.30 & 2.90 & 83.33 & 48.25 & 3.25 & 9.35 & 31.30 & 49.66 \\
\hline & GT-10 & 39.67 & 87.67 & 5.05 & 4.93 & 84.52 & 2.98 & 82.33 & 51.71 & 3.34 & 10.00 & 32.83 & 50.74 \\
\hline \multicolumn{14}{|c|}{ Female/ Line } \\
\hline & IVT-1 & 45.00 & 85.00 & 4.34 & 3.67 & 78.75 & 2.06 & 109.67 & 53.01 & 3.37 & 8.10 & 28.85 & 48.71 \\
\hline & IVT-6 & 41.67 & 102.33 & 4.21 & 3.41 & 84.62 & 2.39 & 97.33 & 46.40 & 3.34 & 9.27 & 29.95 & 48.17 \\
\hline & IVT-8 & 45.00 & 78.00 & 3.68 & 4.33 & 77.54 & 2.27 & 114.33 & 53.25 & 3.48 & 8.35 & 31.51 & 46.47 \\
\hline & IVT-14 & 41.00 & 100.67 & 3.65 & 3.13 & 85.07 & 2.91 & 94.67 & 48.19 & 3.24 & 11.02 & 28.76 & 48.57 \\
\hline & IVT-15 & 40.00 & 104.33 & 3.21 & 2.67 & 80.15 & 2.78 & 105.00 & 54.91 & 3.39 & 10.55 & 28.46 & 46.20 \\
\hline & IVT-16 & 40.67 & 101.67 & 4.05 & 3.23 & 88.37 & 2.97 & 81.67 & 58.42 & 3.57 & 11.71 & 26.19 & 47.67 \\
\hline & IVT-18 & 46.33 & 89.33 & 3.81 & $\mathbf{3 . 8 3}$ & 87.62 & 2.85 & 86.00 & 54.95 & 3.46 & 10.80 & 28.72 & 48.30 \\
\hline & IVT-19 & 41.67 & 113.67 & 3.76 & 3.76 & 85.34 & 2.66 & 94.33 & 53.23 & 3.26 & 11.54 & 33.17 & 48.33 \\
\hline & IVT-20 & 43.33 & 89.67 & 3.34 & 3.03 & 82.48 & 2.49 & 109.00 & 50.39 & 3.48 & 9.81 & 27.99 & 46.97 \\
\hline \multicolumn{2}{|c|}{ Parents mean } & 42.48 & 90.84 & 4.01 & 3.63 & 83.42 & 2.66 & 97.43 & 51.69 & 3.32 & 9.41 & 29.86 & 48.08 \\
\hline \multicolumn{2}{|c|}{ Male mean } & 41.25 & 84.53 & 4.44 & 3.77 & 82.44 & 2.90 & 101.00 & 49.53 & 3.24 & 7.83 & 30.82 & 49.31 \\
\hline \multicolumn{2}{|c|}{ Female mean } & 42.97 & 93.37 & 3.83 & 3.57 & 83.82 & 2.55 & 96.00 & 52.56 & 3.36 & 10.04 & 29.48 & 47.59 \\
\hline \multicolumn{2}{|l|}{ Range } & $\begin{array}{c}39.67- \\
46.33\end{array}$ & $\begin{array}{l}69.00- \\
113.67\end{array}$ & $3.21-5.05$ & $2.81-4.63$ & $\begin{array}{l}77.54- \\
88.37\end{array}$ & $2.06-2.98$ & $\begin{array}{l}81.67- \\
114.33\end{array}$ & $\begin{array}{l}46.40- \\
58.42\end{array}$ & 2.96-3.57 & $\begin{array}{l}8.10- \\
11.71\end{array}$ & $\begin{array}{c}26.19- \\
33.17\end{array}$ & $\begin{array}{l}46.20- \\
50.74\end{array}$ \\
\hline
\end{tabular}


Table.3 Analysis of variance for combining ability of various quantitative characters

\begin{tabular}{|c|c|c|c|c|c|c|c|c|c|c|c|c|c|}
\hline SOURCE & DF & $\begin{array}{c}\text { Days to } \\
50 \% \\
\text { flowering }\end{array}$ & $\begin{array}{c}\text { Plant } \\
\text { height } \\
(\mathrm{cm})\end{array}$ & $\begin{array}{c}\text { No. of } \\
\text { primary } \\
\text { branches } \\
\text { per plant }\end{array}$ & $\begin{array}{c}\text { No. of } \\
\text { secondary } \\
\text { branches } \\
\text { per plant }\end{array}$ & $\begin{array}{c}\text { No. of } \\
\text { capsules } \\
\text { per plant }\end{array}$ & $\begin{array}{c}\text { Capsule } \\
\text { length } \\
(\mathrm{cm})\end{array}$ & $\begin{array}{c}\text { Days to } \\
\text { maturity }\end{array}$ & $\begin{array}{l}\text { No. of } \\
\text { seeds per } \\
\text { capsule }\end{array}$ & $\begin{array}{c}1000 \\
\text { seed } \\
\text { weight } \\
(\mathrm{g})\end{array}$ & $\begin{array}{c}\text { Seed } \\
\text { yield } \\
\text { per } \\
\text { plant }\end{array}$ & $\begin{array}{c}\text { Harvest } \\
\text { index }(\%)\end{array}$ & $\begin{array}{c}\text { Oil } \\
\text { conten } \\
\text { t }(\%)\end{array}$ \\
\hline $\begin{array}{c}\text { REPLICA } \\
\text { TION }\end{array}$ & 2 & 19.43 & 74.03 & 0.01 & 0.32 & 12.32 & $0.02 *$ & 8.57 & $146.16^{* *}$ & 0.02 & $0.23 * *$ & 4.77 & 0.06 \\
\hline LINES & 9 & $42.12 * *$ & $782.45^{* *}$ & $10.36 * *$ & $3.88 * *$ & $342.74 * *$ & $0.47 * *$ & $237.75^{* *}$ & $204.33 * *$ & $0.71 * *$ & $31.36 * *$ & $164.70 * *$ & $6.64 * *$ \\
\hline TESTERS & 3 & 3.47 & $348.63 * *$ & $7.88 * *$ & $1.23 * *$ & 5.66 & $0.15 * *$ & $52.74 * *$ & $52.10 *$ & $0.28 * *$ & $0.95 * *$ & 26.21 & $3.95^{*}$ \\
\hline $\begin{array}{c}\text { LINES X } \\
\text { TESTERS }\end{array}$ & 27 & $13.38 * *$ & $341.37 * *$ & $5.97 * *$ & $1.40 * *$ & $132.80 * *$ & $0.22 * *$ & $139.21 * *$ & $74.98 * *$ & $0.10^{*}$ & $0.95 * *$ & $65.54 * *$ & $3.61 * *$ \\
\hline ERROR & 78 & 3.70 & 28.41 & 0.09 & 0.11 & 10.73 & 0.01 & 7.84 & 18.25 & 0.06 & 0.02 & 10.66 & 1.11 \\
\hline \multicolumn{2}{|l|}{$\sigma_{\mathrm{f}}^{2}$} & $2.39 * *$ & $36.76^{* *}$ & $0.37 * *$ & $17.50 * *$ & $0.02 * *$ & $8.21 * *$ & $10.78 * *$ & $0.05 * *$ & $2.53 * *$ & $8.26 * *$ & $0.25 * *$ & $0.16^{* *}$ \\
\hline \multicolumn{2}{|l|}{$\sigma_{\mathrm{m}}^{2}$} & -0.33 & $0.24 * *$ & $0.06 * *$ & -4.24 & $0.00 * *$ & $-2.88 * *$ & $-0.76^{*}$ & $0.01 * *$ & $0.00 * *$ & -1.31 & $0.01 *$ & -0.02 \\
\hline \multicolumn{2}{|l|}{$\sigma^{2}{ }_{\mathrm{gca}}$} & 0.45 & $10.67 * *$ & $0.15 * *$ & 1.97 & $0.02 * *$ & $0.29 * *$ & $2.53 *$ & $0.02 * *$ & $0.72 * *$ & 1.42 & $0.08 *$ & 0.03 \\
\hline \multicolumn{2}{|l|}{$\sigma_{\text {sca }}^{2}$} & $3.23 * *$ & $104.32 * *$ & $1.96 * *$ & $40.69 * *$ & $0.07 *$ & $43.79 * *$ & $18.91 * *$ & $0.01 * *$ & $0.31 * *$ & $18.30 * *$ & $0.83 * *$ & $0.12 * *$ \\
\hline \multicolumn{2}{|c|}{$\sigma_{\mathrm{gca}}^{2} / \sigma_{\mathrm{sca}}^{2}$} & 0.14 & 0.10 & 0.08 & 0.05 & 0.26 & 0.01 & 0.13 & 2.36 & 1.35 & 0.08 & 0.10 & 0.27 \\
\hline
\end{tabular}

$*, * *$ Significant at $5 \%$ and $1 \% ; \sigma_{\mathrm{f},}^{2} \sigma_{\mathrm{m}, \sigma_{\mathrm{gca}}, \sigma^{2} \mathrm{sca}}=$ female, male, $g c a$ and $s c a$ variance, respectively. d.f. $=$ degree of freedom 
Table.4 General combining ability effects for parents in respect of twelve quantitative characters in sesame ( esamum indicum L.)

\begin{tabular}{|c|c|c|c|c|c|c|c|c|c|c|c|c|}
\hline Parent & $\begin{array}{c}\text { Days to } 50 \\
\text { per cent } \\
\text { flowering }\end{array}$ & $\begin{array}{c}\text { Plant } \\
\text { height } \\
(\mathbf{c m})\end{array}$ & $\begin{array}{c}\text { No. of } \\
\text { Primary } \\
\text { branches } \\
\text { per plant }\end{array}$ & $\begin{array}{c}\text { No. of } \\
\text { Secondary } \\
\text { branches } \\
\text { per plant }\end{array}$ & $\begin{array}{c}\text { No. of } \\
\text { capsules } \\
\text { per plant }\end{array}$ & $\begin{array}{c}\text { Capsule } \\
\text { length } \\
(\mathrm{cm})\end{array}$ & $\begin{array}{c}\text { Days to } \\
\text { maturity }\end{array}$ & $\begin{array}{c}\text { No. of } \\
\text { Seeds } \\
\text { per } \\
\text { capsule }\end{array}$ & $\begin{array}{c}1000- \\
\text { seeds } \\
\text { weight } \\
\text { (g) }\end{array}$ & $\begin{array}{l}\text { Yield per } \\
\text { plant (g) }\end{array}$ & $\begin{array}{c}\text { Harvest } \\
\text { index } \\
(\%)\end{array}$ & $\begin{array}{c}\text { Oil } \\
\text { content } \\
(\%)\end{array}$ \\
\hline IVT-1 & $-2.38 * *$ & $-10.71 * *$ & $-1.50 * *$ & $-0.221 *$ & $3.60 * *$ & $0.15 * *$ & $1.76^{* *}$ & $7.42 * *$ & -0.05 & $-0.90 * *$ & $4.15 * *$ & $-0.52 *$ \\
\hline IVT-6 & -0.71 & $3.21 *$ & $-0.73 * *$ & $-0.636 * * *$ & $1.84^{* *}$ & $0.24 * *$ & -0.99 & $2.98 * *$ & $0.16^{* *}$ & $0.29 * *$ & 0.54 & $-0.59 *$ \\
\hline IVT-8 & -0.88 & $-2.88 *$ & $-0.67 * *$ & $-0.538 * * *$ & $-3.36 * *$ & $-0.04 *$ & 0.26 & -0.81 & $-0.13^{*}$ & $-0.93 * *$ & $-5.28 * *$ & $-1.29 * *$ \\
\hline IVT-13 & $-2.54 * *$ & $-9.54 * *$ & 0.04 & $0.635^{* * *}$ & $8.31 * *$ & $0.12 * *$ & -0.16 & $4.48 * *$ & $0.17^{* *}$ & $-0.37 * *$ & $-5.21 * *$ & -0.47 \\
\hline IVT-14 & $2.04 * *$ & $10.96 * *$ & $-0.55^{* *}$ & $-0.765 * * *$ & $1.50 * *$ & $-0.18 * *$ & $-2.91 * *$ & $-2.17 *$ & -0.02 & $-0.25 * *$ & $-4.44 * *$ & 0.40 \\
\hline IVT-15 & $0.96^{*}$ & $7.13^{* *}$ & $1.05^{* *}$ & $0.358 * * *$ & $-9.53 * *$ & $0.14^{* *}$ & -0.66 & $-4.58 * *$ & $-0.25 * *$ & $-0.35^{* *}$ & $1.66^{*}$ & $0.55^{*}$ \\
\hline IVT-16 & $-1.46 * *$ & $-6.79 * *$ & $1.17 * *$ & $-0.337 * * *$ & $-4.31 * *$ & $0.17 * *$ & $-5.58 * *$ & $-4.15 * *$ & $0.19 * *$ & $-0.57 * *$ & $3.19 * *$ & $0.50 *$ \\
\hline IVT-18 & $2.46^{* *}$ & $6.46^{* *}$ & 0.14 & $0.318 * *$ & $-1.66 * *$ & $-0.32 * *$ & $2.51 * *$ & $2.80 * *$ & -0.11 & $-0.27 * *$ & $3.04 * *$ & -0.14 \\
\hline IVT-19 & 0.29 & $8.21 * *$ & $1.30 * *$ & $0.852 * * *$ & $0.53 * *$ & -0.03 & $10.34 * *$ & $-3.21 * *$ & $0.44 * *$ & $4.45 * *$ & $2.94 * *$ & 0.28 \\
\hline IVT-20 & $2.21 * *$ & $-6.04 * *$ & $-0.25 * *$ & $0.335^{* * *}$ & $6.76^{* *}$ & $-0.26^{* *}$ & $-4.58 * *$ & $-2.77 * *$ & $-0.39 * *$ & $-1.10^{* *}$ & -0.58 & $1.29 * *$ \\
\hline TKG-21 & 0.14 & $1.99 * *$ & $-0.44 * *$ & $-0.136^{*}$ & -0.10 & $0.02 *$ & 0.38 & $-1.35^{*}$ & $0.12 * *$ & $-0.22 * *$ & 0.16 & -0.07 \\
\hline TKG-22 & 0.34 & $-2.28 * *$ & $-0.23 * *$ & $0.246^{* * *}$ & -0.33 & $-0.10 * *$ & -0.56 & $1.46^{*}$ & $-0.08 * *$ & $-0.05 * *$ & $1.10^{* *}$ & -0.24 \\
\hline GT-2 & -0.02 & $3.72 * *$ & -0.07 & 0.084 & -0.35 & $0.06^{* *}$ & $1.64 * *$ & 0.74 & $-0.08 * *$ & $0.09 * *$ & -0.08 & $0.53^{* *}$ \\
\hline $\begin{array}{c}\text { CD 95\% } \\
\text { GCA(Line) }\end{array}$ & 0.87 & 0.72 & 0.974 & 1.097 & 0.29 & 0.59 & 1.478 & 0.118 & 1.287 & 0.273 & 0.126 & 0.575 \\
\hline $\begin{array}{c}\text { CD 95\% } \\
\text { GCA(Teste) }\end{array}$ & 0.711 & 0.588 & 0.795 & 0.896 & 0.237 & 0.482 & 1.207 & 0.096 & 1.051 & 0.223 & 0.103 & 0.469 \\
\hline $\mathrm{N}+\mathrm{ve}$ & 4 & 7 & 4 & 6 & 7 & 4 & 5 & 5 & 4 & 5 & 6 & 4 \\
\hline $\mathrm{N}$-ve & 3 & 7 & 7 & 7 & 5 & 4 & 6 & 5 & 10 & 4 & 4 & 3 \\
\hline
\end{tabular}

Table.5 Performance of promising hybrids for seed yield per plant

\begin{tabular}{|c|c|c|c|c|c|}
\hline $\begin{array}{l}\text { Best specific } \\
\text { combination }\end{array}$ & Per se performance & $\begin{array}{c}\text { sca } \\
\text { effects }\end{array}$ & Heterobeltiosis & $\begin{array}{l}\text { gca effects of the } \\
\text { parents involved }\end{array}$ & $\begin{array}{l}\text { Significant heterosis for other } \\
\text { traits over better parent }\end{array}$ \\
\hline IVT-19 X GT-2 & 15.59 & $1.25 * *$ & 33.39** & GX G & Harvest index \\
\hline IVT-6 X GT-10 & 11.28 & $1.21 * *$ & $21.75 * *$ & GX G & Days to maturity. \\
\hline IVT-13 X TKG-21 & 9.84 & $0.83 * *$ & 19.39* & $\mathbf{P} \mathbf{X} \mathbf{A}$ & $\begin{array}{c}\text { Plant height, } \\
\text { 1000-seed weight }\end{array}$ \\
\hline
\end{tabular}


Based on seed yield per plant, top three crosses shows high sca effects along with their per se performance and heterobeltiotic effect having best specific combination for seed yield per plant were obtained either through average $\mathrm{x}$ average, average $\mathrm{x}$ poor and poor $\mathrm{x}$ poor parental combination. The best specific combination viz., IVT-19 X GT2 recorded the desirable significant sca effects for traits of seed yield per plant and harvest index. The second best cross i.e., IVT-6 X GT-10 had desirable significant sca effects for plant height and seed yield per plant. Whereas the third best cross IVT-13 X TKG21 had significant $s c a$ effects for plant height, 1000-seed weight and seed yield per plant. These results are getting support from the findings of Solanki and Singh (2006), Sharmila and Ganesh (2008), Toprope (2008), Bharathi Kumar and Vivekanandan (2009), Praveenkumar (2009), Yamanuraet al., (2009), Senthil Kumar and Kannan (2010) and RanjithRajaram and Senthil Kumar (2011).

In the present investigation it would be concluded that, the parental genotypes viz., IVT-6, IVT-19, GT-2 and GT-10 were found good general combiners and exhibited the positive and significant gca for seed yield per plant. These parents could be used in the breeding programme to improve seed yield and its component characters. It may be inferred that the genotypes can maintain their superiority in per se performance and also combining ability effects. The crosses, IVT19 X GT-2, IVT-6 X GT-10, and IVT-13 X TKG-21 recorded desirable SCA effects and significant positive heterosis for seed yield per plant and most studied traits which were controlled by non-additive gene effects. Under such situation, it would be worthwhile to resort by using breeding methodologies such as biparental mating or few cycles of recurrent selection followed by pedigree selection may fruitful results.

\section{References}

Anuradha T. and Reddy L.K. 2008. Nature of gene action for quantitative traits in sesame. Agricultural Science Digest 28 (4): 307-308.

Ahmed S.B.M. and Adam S.I. 2014. Combining ability for yield and yield components in six parents and their 15 F1 hybrids of sesame (Sesamum indicum L.) in half diallel mating design. J. Plant Breed. Crop Sci., 6(12): 179-180

FAOSTAT 2015. Food and Agricultural Organization of the United Nations

Kempthorne O. 1957. An Introduction to Genetic Statistics. John Wiley and Sons, Inc., New York.

Panse V.G. and Sukhatme P.V. 1978. Statistical methods for agriculture Workers I.C.A.R., New Delhi.

Dhillon B.S. 1975. The application of partialdiallel crosses in plant breeding-A review. Crop Improv., 2: 1-7.

Allard R.W. 1960. Principles of plant breeding. John Wiley and Sons, Inc., New York, London.

Senthilkumar P. and Ganesan J. 2001. Combining ability in sesame (Sesamum indicum L.). In: Natl. Sem. on Sesame Crop Improvement and its Future Prospects. Annamalai Univ., 28th February and 1st March, p. 9.

Yamanura., Madhusudan K., and Nadaf H.L. 2009, Combining ability and gene action for yield and yield components in sesame (Sesamum indicum L.). Karnataka Journal of Agricultural Sciences, 22 (2): 11-17

Saravanan T., Thirugnana S.K., and Ganesan J. 2000a. Combining ability and heterosis for earliness characters in sesame (Sesamum indicum L.), Sesame and Safflower Newsletter, 15: 7-13

Preveenkumar K., Madhusudan H., Nadaf L., Patil R.K., and Deshpande S.K. 2012. 
Combining ability and gene action studies in inter-mutant hybrids of sesame (Sesamum indicum L.). Karnataka Journal of Agricultural Science, 25(1): 1-4.

Singh A.K. 2007. Heterosis in relation to combining ability for yield and its components in sesame (Sesamum indicum L.). Journal of Oilseeds Research, 24(1): 51-55.

Kavitha M., Sethupathi R.R., and Raveendran T.S. 1999. Combining ability in sesame (Sesamum indicumL.). Journal of Oilseeds Research, 16: 27-31

Thakare V.V., Parde S.B., Pande M.K., Lahane P.S., and Peshattiwar P.D. 1999. Combining ability studies in Sesame. Journal Maharashtra Agricultural University, 24: 256-259.

Manivaran N. and Ganesan J. 2001. Line x Tester analysis in sesame (Sesamum indicum L.). Indian J. Agric. Res., 35: 90-94

Vidhyavathi R., Manivannan N., and Muralidharan V. 2005. Line x tester analysis in sesame (Sesamum indicum L.). Indian J. Agric. Res., 39: 225-228

Thiyagu K., Kandasamy G., Manivannan N., and Muralidharan V. 2007. Studies in combining ability for economic traits in cultivated sesame (Sesamum indicum L.). Madras Agricultural Journal, 94(712): 168-173.

Bharathi K. and Vivekanandan P. 2009. Studies on combining ability studies in sesame (Sesamum indicum L.).Electron. J. Plant Breed., 1: 33-36

Mothilal A. and Ganesan K.N. 2005. Heterosis studies in sesame (Sesamum indicum L.). Agricultural Science Digest., 25(1): 74-76.

Azeez M.A. and Morakinyo J.A. 2014. Combining ability and potential of oil quality improvement in sesame (Sesamum indicum L.). J.
Agroalimentary Processes and Technologies., 20(1): 1-8.

Mishra H.P., Mishra RC., and Sahu P.K. 2009. Combining ability and nature of gene action in Sesame (Sesamum indicum L.). Indian J. Agric. Res., 43(2): 119-123.

Kumar K.B. and Vivekanandan P. 2009. Studies on combining ability studies in sesame (Sesamum indicum L.). Elect. J. Plant Breed., 1: 33-36

Krishnaiah G., Reddy K.R., Reddy G.L.K., and Sekhar M.R. 2002. Combining ability in sesame. Crop. Res. (Hisar)., 24(1): 72-76.

Devasena V., Muralidharan V., and Puniyha D. 2001. Studies on combining ability for yield related traits in sesame (Sesamum indicum L.). Research on Crops, 2(3): 409-413.

Prajapati K.P., Patel K.M., Prajapati B.H. and Patel C.J. 2006. Genetic analysis of quantitative traits in sesame (Sesamum indicum L.). J. Oilseeds Res., 23(2): 171-173.

Toprope V.N. 2008. Heterosis in relation to combining ability for yield and its contributing traits in sesame (Sesamum indicum L.). J. Oilseeds Res., 25(1): 7981.

Prajapati K.P., Patel K.M., Prajapati B.H., and Patel C.J. 2006. Genetic analysis of quantitative traits in sesame (Sesamum indicum L.). J. Oilseeds Res., 23(2): 171- 173.

Sankar P.D. and Kumar C.R.A. 2001. Heterosis for yield and yield components in sesame (Sesamum indicum L.). Sesame and Safflower Newsletter, 16: 6-8.

Kar, U.C., D. Swain and J. R. Mahapatra, 2002. Hybrid performance in relation to combining ability for seed yield and its components in sesame (Sesamum indicum L.) Research on Crops, 2(1): 103-109. 
Hamouda, M.A.A., 2001. Diallel analysis for some agronomic and quality characters in sesame (Sesamum indicum L.). M. Sc. Thesis, Fac. of Agric., Zagazig Univ., Egypt.

Hoballah, A.A., A.A. Kandil and M.N. Kandil, 2001. Diallel analysis for studying heterosis, combining ability and nature of gene action in sesame (Sesamum indicum L.). The Second Plant Breed. Conf. Oct. $2^{\text {nd }} 2001$ (Assiut University, Egypt).

Krishna Devi, M., S. Tnirugnana Kumar and J. Ganesan, 2002. Combining ability and heterosis for reproductive efficiency in sesame (Sesamum indicum L.). Sesame and Safflower Newsletter, 17: 5-9.

Saravanan, S. and N. Nadarajan, 2003. Combining ability studies in sesame. Crop Res., 25(2): 319-324.

Sanker, P.D. and C.R.A. Kummar, 2003. Genetic analysis of yield and related components in sesame (Sesamum indicum L.). Crop Res. (Hisar) 25(1): 91-95.

Abd Elaziz, Ghada B., A.A. Abu El-Ezz and Samar A.M. El-Shakhess, 2010. Heterosis and combining ability for seed yield and its components in sesame under stress conditions. Egypt. J. Plant Breed, 14(2): 59-70.

Sedeck, F.Sh. and Wafaa W.M. Shafie, 2013. Estimates of gene action and interrelationships among yield characters in diallel crosses of sesame (Sesamum indicum L.). Assiut J. Agric. Sci., 44(3): 15-31.

Yermanos, D.M., 1980. Hybridization of Crop Plants. American Society of Agronomy and Crop Science Society of America Publishers Madison, Wisconsin, USA, pp: 549-563.

Griffing, B., 1956. Concept of general and specific combining ability in relation to diallel crossing system. Aust. J. Bio. Sci., 9: 463-493.

Acevedo, B.M.A. and E. J. MonteverdePenso, 1998. Comparison of two crosses design through estimates of genetic variance in a population of indehiscent African type of sesame. Agronomia Tropical (Maracay), 48(4): 441-456. [C.F. Plant Breed. Abst. 69(8): 8090.

Krihnaiah, G., K.R. Reddy, G.L.K. Reddy and M.R. Sekhar, 2002. Combining ability in sesame. Crop Res. (Hisar), 24(1): 7276.

\section{How to cite this article:}

Kanak Saxena and Rajani Bisen. 2017. Line x Tester Analysis in Sesame (Sesamum indicum L.). Int.J.Curr.Microbiol.App.Sci. 6(7): 1735-1744. doi: https://doi.org/10.20546/ijcmas.2017.607.209 